\title{
LASER DRIVEN ELECTRON ACCELERATION TO GeV ENERGIES IN PLASMA CHANNELS*
}

\author{
P. Sprangle ${ }^{\#}$, B. Hafizi ${ }^{+}$, A. Ting, C.I. Moore, R. F. Hubbard and A. Zigler* \\ Plasma Physics Division, Naval Research Laboratory, Washington, DC 20375
}

\begin{abstract}
This paper presents a brief discussion of ultra-short laser pulse propagation and laser wakefield acceleration in plasma channels. An envelope equation for the perturbed laser beam radius is given, including finite-pulse length and nonlinear effects. Angular distribution of electrons generated by the process of laser ionization and ponderomotive acceleration (LIPA) is also discussed. An example of laser wakefield acceleration to electron energies greater than $1.2 \mathrm{GeV}$ in a preformed plasma channel is described.
\end{abstract}

\section{INTRODUCTION}

Electron acceleration in a laser wakefield is limited by a number of processes, including electron slippage and laser beam diffraction. To reduce the effects of diffraction the laser pulse can be propagated in a preformed plasma channel having a suitable (parabolic) radial density profile. In plasma channels propagation distances much longer than a vacuum Rayleigh range, $Z_{\mathrm{R} 0}=\pi r_{0}{ }^{2} / \lambda$, can be achieved [1-3]. Here, $r_{0}$ is the laser spot size and $\lambda$ is the vacuum wavelength. Finite pulse length effects can modify the propagation dynamics in long channels. Short pulse effects lead to envelop modulation, damping due to phase mixing, and group velocity dispersion [3,4]. An envelope equation for the perturbed pulse envelope is presented which includes these effects along with nonlinearity [3]. A second topic discussed is the generation of electrons by laser ionization and ponderomotive acceleration (LIPA) [5]. By means of LIPA electrons can be stripped from atoms in the region of high laser intensity and accelerated by the ponderomotive force. Finally, an example of laser wakefield acceleration of electrons to $1.2 \mathrm{GeV}$ in a $15-\mathrm{cm}$ long plasma channel is described.

\section{ENVELOPE EQUATION}

An envelope equation for the radius of the laser pulse propagating in a channel can be obtained by assuming the slowly-varying form for the electric field, $\boldsymbol{E}=\left(E_{0} / 2\right)$ $\exp [\mathrm{i}(k z-\omega t)] \boldsymbol{e}_{\mathrm{x}}+$ c.c., where $E_{0}(\boldsymbol{r}, t)$ is the amplitude, $k$ is the wavenumber, $\omega=2 \pi c / \lambda$ and $c$ is speed of light in

\footnotetext{
*Work supported by DoE \& ONR. The authors acknowledge the assistance of J. Penano in preparing the computer graphics.

\# Email:sprangle@ppd.nrl.navy.mil

${ }^{+}$Icarus Research, Inc. PO Box 30780, Bethesda, MD 20824-0780

* Hebrew University, Jerusalem, Israel
}

vacuo. The amplitude $E_{0}$ is assumed to be expressible as $E_{0}=b \exp \left[\mathrm{i} \phi-(1-\mathrm{i} \theta) r^{2} / r_{\mathrm{s}}^{2}\right]$, where $b$ is the amplitude, $\phi$ is the phase, $\theta$ is related to the wavefront curvature and $r_{\mathrm{s}}$ is the spot size. The variables $b, \varphi, \theta, r_{s}$ are taken to be real and slowly varying functions of $z$ and $t$. The radial dependence of the plasma frequency is given by

$$
\omega_{p}(r)=\left[1+\left(\Delta n / n_{p o}\right)\left(r / r_{c}\right)^{2}\right]^{1 / 2}
$$

where $n_{p o}$ is the on axis density, $n_{p o}+\Delta n$ is the density at the edge of the plasma channel $\left(r=r_{\mathrm{c}}\right), \omega_{\mathrm{p} 0}=$ $\left(4 \pi n_{\mathrm{p} 0} q^{2} / m\right)^{1 / 2}$ is the plasma frequency on axis, $q$ is the charge and $m$ is the mass of an electron. The focusing parameter associated with the channel is $K_{\mathrm{c}}=$ $\left(\omega_{\mathrm{p} 0} / c\right)\left(\Delta n / n_{\mathrm{p} 0}\right)^{1 / 2}$. Making use of the source-dependent expansion approach [6], the equilibrium radius is found to be given by $r_{\mathrm{eq}}=r_{\mathrm{m}}(1-P)^{1 / 4}$, where $r_{\mathrm{m}}=\left(2 r_{\mathrm{c}} / K_{\mathrm{c}}\right)^{1 / 2}$ is the matched beam radius, $P$ is the laser power normalized to the critical power for focusing, $r_{\mathrm{c}}$ is radius of the parabolic channel and $K_{\mathrm{c}}$ is the on axis plasma wavenumber [3]. In the limit $P<<1$ the envelope equation for the perturbed laser pulse radius, normalized to $r_{0}$, and is given by

$$
\frac{\partial^{2} \delta R}{\partial Z^{2}}+\frac{4}{n^{2}} \delta R-\frac{4 \varepsilon}{n^{2}} \frac{\partial^{2} \delta R}{\partial Z \partial \zeta}+\frac{32 \varepsilon \zeta}{n^{2}} \frac{\partial \delta R}{\partial Z}=0,
$$

where $Z=z / Z_{\mathrm{R} 0}, \zeta=(z-c n t) / l_{0}, n=c k / \omega$ is the refractive index, an initial axial profile $b(z=0) \sim \exp (-$ $\left.4 \zeta^{2} / l_{0}^{2}\right)$ has been assumed and $\varepsilon=\lambda /\left(2 \pi l_{0}\right) \ll<1$. The solution to this equation is

$$
\delta R=\delta R_{0} \cos (2 Z / n) \exp \left(-16 \varepsilon \zeta Z / n^{2}-16 \varepsilon^{2} Z^{2} / n^{4}\right),
$$

where $\delta R_{0}$ is a constant. The first term in the exponential leads to modulation of the pulse, with the back of the pulse $(\zeta<0)$ growing and the front $(\zeta>0)$ damped. The second term in the exponential is due to phase mixing and leads to damping of the perturbation.

The modulation and damping predicted by the envelope equation has been observed in a 2-D simulation of laser 
propagation in a channel. At early times, $\delta R$ should exhibit growth or damping at the normalized linear rate $\Gamma(\zeta)=-16 \varepsilon \zeta / n^{2}$. The simulation rate $\Gamma$ is determined from a semi-logarithmic plot of $\delta R$ against $z$, fitting the peaks of the oscillations. The laser parameters were $\lambda=0.8 \mu \mathrm{m}, I_{0}=24 \mu \mathrm{m}, r_{0}=14.5 \mu \mathrm{m}$, and $Z_{R}=0.083$ $\mathrm{cm}$. Figure 1 is a plot of $\Gamma(\zeta)$ (diamonds) from simulation using the code described in [2]. The straight line is the analytical growth or damping rate. As expected, the front of the beam is damped, while the back exhibits growth in $\delta R$. The simulation results consistently lie below the theoretical growth rate. This is due to the phase mix damping contained in the last term in the $\delta R$ equation. The long time behavior in the simulations is eventually dominated by the phase mixing term and exhibits the expected damping in $z^{2}$.

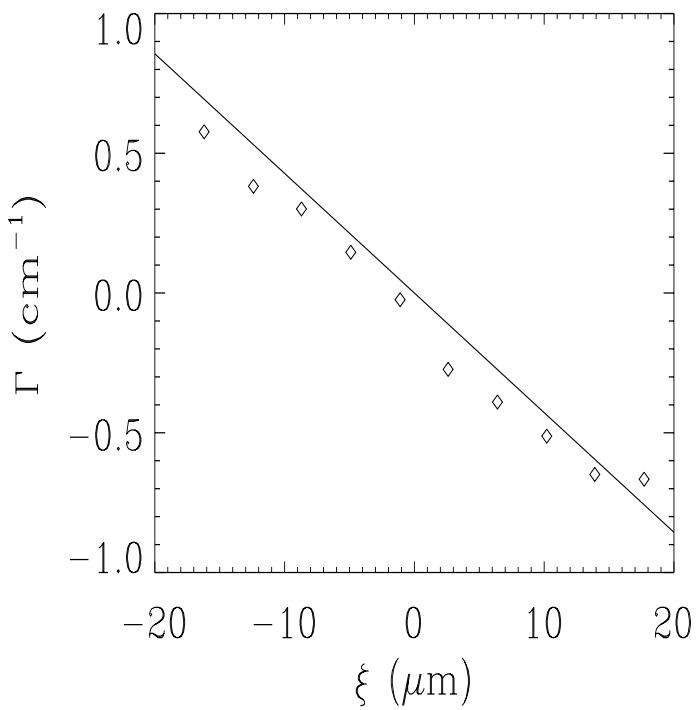

Fig. 1. Linear growth or damping rate $\Gamma$ versus position $\zeta$ within the pulse. The diamonds are from the simulation code, and the solid line is from the analytical solution for $\delta R$.

In the high power limit, $P \leq 1$, there are additional contributions to the envelope equation, arising from nonlinearities such as the relativistic focusing effect [3]. Short pulse effects lead to substantial modification of the nonlinear focusing processes. Figure 2 is a surface plot of the spot size $R$ as a function of $\zeta / \lambda$ and $Z$, with (a) finite pulse length effects $(\varepsilon \neq 0)$ and (b) finite pulse length effects neglected $(\varepsilon=0)$. The parameters are $\lambda=1 \mu \mathrm{m}$, $l_{0}=20 \mu \mathrm{m}$ and $P=0.56$. This plot shows the envelope modulation and significant enhancement of nonlinear focusing.

\section{LASER IONIZATION AND PONDEROMOTIVE ACCELERATION}

In tunneling ionization the electric field of the laser depresses the Coulomb barrier in the atom allowing an electron to tunnel through. When an electron is released from an atom in the presence of the laser field the ponderomotive force can accelerate it to relatively high energies depending on the laser intensity. Laser acceleration and ponderomotive acceleration (LIPA) [5] can be a source of high brightness electrons for injection into laser driven accelerators. In LIPA, the electrons are ejected from essentially a point source and are highly collimated when the laser beam is linearly polarized [5]. This is borne out by recent experimental results at the Naval Research Laboratory [5]. The electrons are ejected into a forward cone with half angle given by

$\vartheta=\tan ^{-1} \sqrt{2 /(\gamma-1)}$,

where $\gamma$ is the relativistic factor of the accelerated electrons.

\section{ELECTRON ACCELERATION IN A PLASMA CHANNEL}

Laser wakefield acceleration in a preformed plasma channel can result in significant energy gain. As an example consider a $1 \mu \mathrm{m}$ wavelength, $l_{0}=50 \mu \mathrm{m}$, circularly polarized laser pulse with spot size $r_{0}=30 \mu \mathrm{m}$ and intensity $2.75 \times 10^{18} \mathrm{~W} / \mathrm{cm}^{2}$ that is matched to a plasma channel with on axis plasma wavelength $2 \pi c / \omega_{\mathrm{p} 0}=75$ $\mu \mathrm{m}$ (on-axis density $2 \times 10^{17} \mathrm{~cm}^{-3}$ ). In this case the peak accelerating gradient associated with the wakefield is $\sim 24$ $\mathrm{GV} / \mathrm{m}$ and the peak transverse electric focusing field is $\sim 5$ $\mathrm{GV} / \mathrm{m}$. Figure 3 is a plot of energy as a function of propagation distance $z$ for an electron that is injected into the wakefield locally; for example by the LIPA process. For this electron, injected on axis behind the laser pulse with axial momentum $0.3 m c$, the plot shows that energies in excess of $1.2 \mathrm{GeV}$ can be reached in a distance $\sim 15 \mathrm{~cm}\left(\sim 54 Z_{\mathrm{R} 0}\right)$. Beyond this length, phase slippage occurs and the energy decreases.

\section{CONCLUSIONS}

This paper presents highlights of a number of interesting results associated with high intensity short pulse lasers. A brief discussion of ultra-short laser pulse propagation and laser wakefield acceleration in preformed plasma channels is given. In particular, an envelope equation for the perturbed laser beam radius, including finite-pulse length and nonlinear effects, is discussed. Angular distribution of electrons generated by the process of laser ionization and ponderomotive acceleration is also mentioned. An example of laser wakefield acceleration to electron energies greater than $1.2 \mathrm{GeV}$ in a preformed plasma channel is described. 

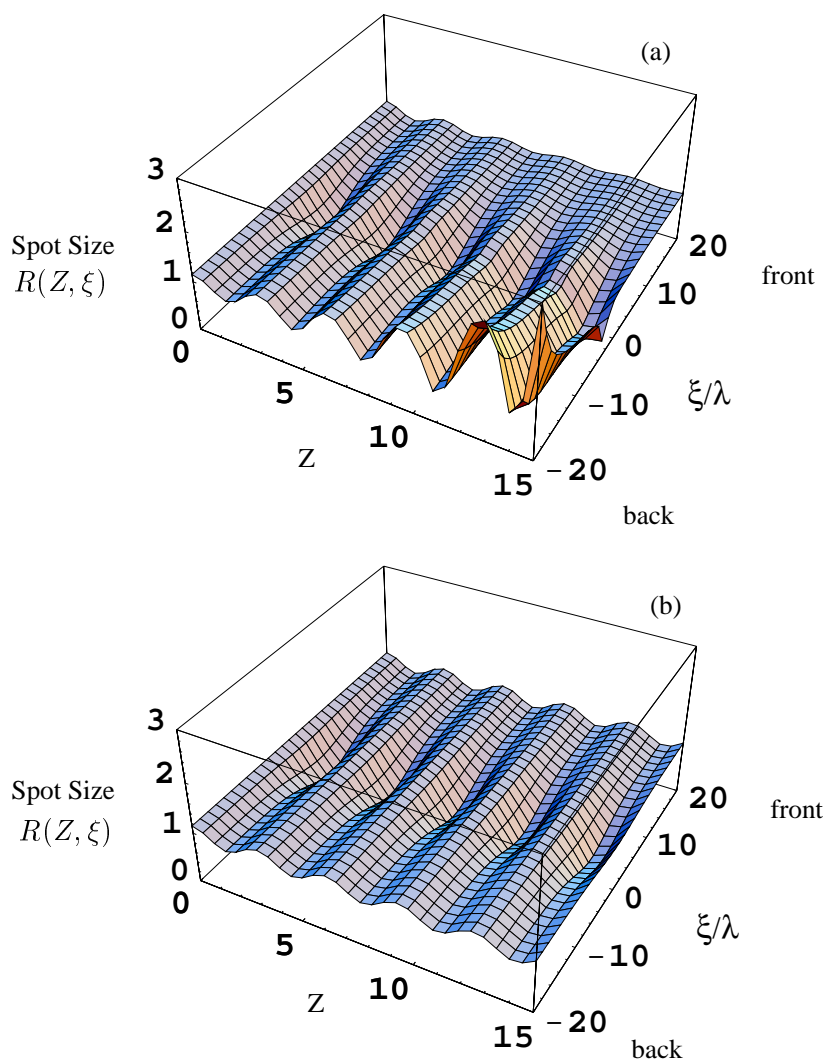

Fig. 2 Surface plot of spot size $R$ as a function of $\zeta / \lambda$ and propagation distance $z$. In (a) finite pulse length effects are include while in (b) finite pulse length effects are neglected.

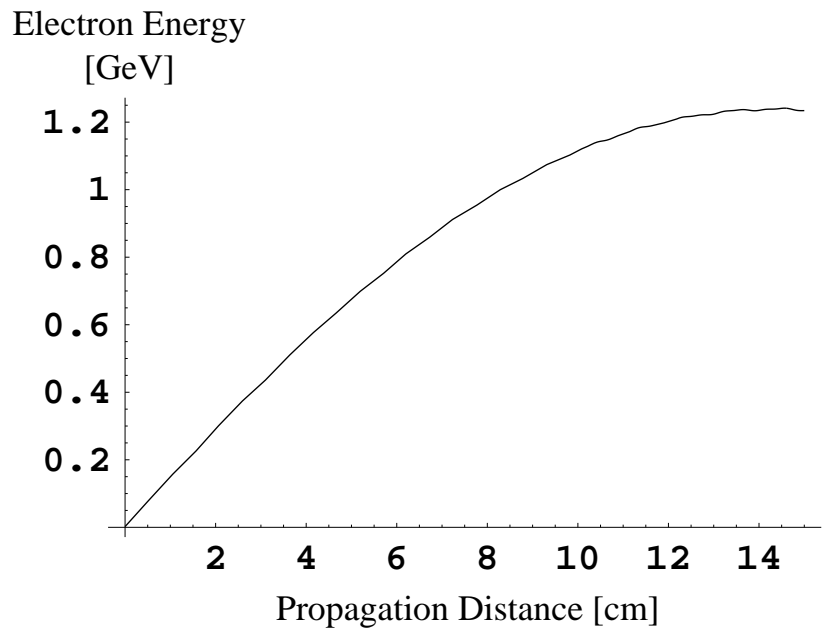

Fig. 3. Plot of electron energy gain due to laser wakefield acceleration versus distance along a preformed plasma channel.

\section{REFERENCES}

[1] H.M. Milchberg, T.R. Clark, C.G. Durfee III, T.M. Antonsen and P. Mora, Phys. Plasmas 3, 2149 (1996)
[2] Y. Ehrlich, C. Cohen, D. Kaganovich, A. Zigler, R.F. Hubbard, P. Sprangle and E. Esarey, J. Opt. Soc. Am. B15, 2416 (1998)

[3] P. Sprangle, B. Hafizi and P. Serafim, Phys. Rev. Lett. 82, 1173 (1999); Phys. Rev. E59, 3614 (1999)

[4] E. Esarey and W.P. Leemans, Phys. Rev. E59, 1082 (1999)

[5] C.I. Moore, A. Ting, S.J. McNaught, J. Qiu, H.R. Burris and P. Sprangle, Phys. Rev. Lett. 82, 1688 (1999)

[6] P. Sprangle, A. Ting and C.M. Tang, Phys. Rev. Lett. 59, 202 (1987); Phys. Rev. A36, 2773 (1987). 